\title{
Leptin Signaling in the Medial Nucleus Tractus Solitarius Reduces Food Seeking and Willingness to Work for Food
}

\author{
Scott E Kanoski*,', Amber L Alhadeff ${ }^{2}$, Samantha M Fortin ${ }^{3}$, Jennifer R Gilbert ${ }^{2}$ and Harvey J Grill ${ }^{2}$ \\ 'Department of Biological Sciences, University of Southern California, Los Angeles, CA, USA; ${ }^{2}$ Department of Psychology, University of \\ Pennsylvania, Philadelphia, PA, USA; ${ }^{3}$ Graduate Program in Neuroscience, University of Illinois at Chicago, Chicago, IL, USA
}

\begin{abstract}
The adipose-derived hormone leptin signals in the medial nucleus tractus solitarius (mNTS) to suppress food intake, in part, by amplifying within-meal gastrointestinal (Gl) satiation signals. Here we show that $\mathrm{mNTS}$ leptin receptor (LepRb) signaling also reduces appetitive and motivational aspects of feeding, and that these effects can depend on energy status. Using the lowest dose that significantly suppressed 3-h cumulative food intake, unilateral leptin $(0.3 \mu \mathrm{g})$ administration to the mNTS (3 h before testing) reduced operant lever pressing for sucrose under increasing work demands (progressive ratio reinforcement schedule) regardless of whether animals were energy deplete (food restricted) or replete (ad libitum fed). However, in a separate test of food-motivated responding in which there was no opportunity to consume food (conditioned place preference (CPP) for an environment previously associated with a palatable food reward), mNTS leptin administration suppressed food-seeking behavior only in chronically food-restricted rats. On the other hand, mNTS LepRb signaling did not reduce CPP expression for morphine reinforcement regardless of energy status, suggesting that mNTS leptin signaling differentially influences motivated responding for food vs opioid reward. Overall results show that mNTS LepRb signaling reduces food intake and appetitive food-motivated responding independent of energy status in situations involving orosensory and postingestive contact with food, whereas food-seeking behavior independent of food consumption is only reduced by mNTS LepRb activation in a state of energy deficit. These findings reveal a novel appetitive role for LepRb signaling in the mNTS, a brain region traditionally linked with processing of meal-related Gl satiation signals.

Neuropsychopharmacology (2014) 39, 605-6I3; doi: I0.1038/npp.2013.235; published online 2 October 2013
\end{abstract}

Keywords: satiation; reward; conditioned place preference; obesity; hindbrain; NTS

\section{INTRODUCTION}

Leptin, secreted primarily from white adipose cells, acts on receptors (leptin receptor, LepRb) in the brain to reduce body weight by suppressing food intake and increasing energy expenditure. Historically, attention has been directed toward leptin's action in the hypothalamus, particularly the arcuate hypothalamic nucleus (ARC). More recent findings reveal that leptin's powerful control over energy balance is anatomically distributed (Grill, 2010; Grill and Kaplan, 1990; Grill and Kaplan, 2002a) as it involves contributions from midbrain and forebrain regions such as the ventral tegmental area (Fulton et al, 2006; Hommel et al, 2006) and the hippocampus (Kanoski et al, 2011) that are associated with the control of motivational, learned, and rewarding aspects of food intake, as well as in extra-ARC hypothalamic nuclei (Leinninger et al, 2009; Zhang et al, 2011) and hindbrain nuclei (Grill et al, 2002b; Huo et al, 2007; Schwartz and Moran, 2002; Skibicka and Grill, 2009)

\footnotetext{
*Correspondence: Dr SE Kanoski, Department of Biological Sciences, University of Southern California, 3560 Watt Way, PED 107, Los Angeles, CA 90089-0652, USA, Tel: +1 213821 5762, Fax: + I 213740 7909, E-mail: kanoski@usc.edu Received 27 July 2013; revised 23 August 2013; accepted 29 August 20।3; accepted article preview online 4 September 2013
}

whose neural processing is typically associated with the control of need-based food intake (ie, feeding driven by energy deficit). Within the hindbrain, the LepRb is most densely expressed in the medial nucleus tractus solitarius (mNTS) of the dorsal vagal complex (Caron et al, 2010; Elias et al, 2000; Huo et al, 2006; Li et al, 1999; Patterson et al, 2011), which is the first CNS site to receive vagal afferentmediated gastrointestinal (GI) signals and is a critical nucleus for satiation signal processing and meal size control (Grill and Hayes, 2009; Schwartz, 2010). Indeed, LepRb signaling in mNTS neurons potently reduces food intake by amplifying the food intake-suppressive effects of various within-meal GI-derived satiation signals, including the intestinally secreted peptide cholecystokinin (Hayes et al, 2010), mechanical distention of the stomach (Huo et al, 2007), and intestinal delivery of a mixed nutrient meal (Kanoski et al, 2012b). These findings have identified mNTS neurons as a key site of integration between leptin signaling and GI satiation signals in the control of meal size (for review, see Grill, 2010; Grill and Hayes, 2012).

Here we examine a novel role for mNTS LepRb signaling in the control of food intake. Experiments described directly test the hypothesis that the anorectic effects triggered by mNTS LepRb signaling are not exclusively mediated through amplification of within-meal GI-derived satiation signals, but also involve the suppression of appetitive and 
motivational aspects of feeding, which include: (a) an animal's willingness to work for palatable food, and (b) food seeking independent of orosensory and/or postingestive contact with food. Leptin or vehicle are infused intra-mNTS before behavioral tests of learned food-motivated responding using a dose of leptin $(0.3 \mu \mathrm{g})$ that was first determined to be the lowest effective dose for significant short-term $(3 \mathrm{~h})$ food intake suppression in free-feeding rats. Willingness to work for food is examined using an operant lever-pressing paradigm with a progressive ratio (PR) reinforcement schedule in which the number of lever presses required to obtain food reinforcement increases exponentially. Food-seeking behavior is assessed using a conditioned place preference (CPP) paradigm in which freely moving rats chose between a location/environment where palatable food (high in saturated fat and sucrose) was previously available for consumption, $v s$ another location where food was neither presented nor consumed.

Leptin's control over energy balance is highly dependent on energy status. This is most clearly supported by the fact that levels of endogenous circulating leptin directly correlate with overall adiposity (Ahima et al, 2000), and by studies showing that the engagement of intracellular signaling pathways following neuronal LepRb activation is disrupted in the pathophysiological state of obesity (Munzberg et al, 2005). Here we determine whether effects of mNTS LepRb activation on food-motivated responding (both for operant responding and CPP) are dependent on energy status by comparing effects in rats whose access to food is chronically restricted (daily rations) $v s$ rats given ad libitum access to food in the home cage throughout the duration of the experiment. In the latter case, reduction of appetitive responding would suggest that mNTS LepRb activation inhibits food-directed behaviors that are driven by factors other than caloric deficit resulting from food restriction.

Both CPP and operant lever-pressing paradigms have been used to evaluate the effects of both drug and food reward. The common neural and neurochemical controls of these food and drug reward-mediated effects continue to attract attention (eg, Kenny, 2011; Tomasi and Volkow, 2013; Volkow et al, 2013a, b). The extent that CNS LepRb signaling reduces appetitive behavior directed toward reinforcers other than food is largely unknown. Here we examine whether mNTS LepRb activation reduces motivated responding for opioid-based reward (CPP for morphine), both in energy-deplete and -replete subjects. The potential for mNTS LepRb signaling to reduce appetitive responding for morphine is supported by findings showing that leptin and opioid agonists (either peripheral or centrally delivered) have opposing effects on mesolimbic dopaminergic signaling (Baldo and Kelley, 2007; De Luca et al, 2011; Fulton et al, 2006; Hommel et al, 2006), suggesting that leptin and morphine may differentially influence motivated behavior by acting on a common neurochemical system. Further, intracerebroventricular leptin administration reduces the food deprivationinduced relapse to heroin seeking (ie, reinstatement of conditioned responding for morphine following extinction) in rats (Shalev et al, 2001).

Overall, our results show that mNTS LepRb signaling reduces appetitive responding for palatable food, and these effects can depend on energy status. The reduction of food-motivated responding by mNTS LepRb activation in the energy replete (nondeprived) state was observed, but only in a test that allowed for consumption of the food reinforcement (operant responding for food reinforcement). By contrast, in the absence of direct contact with food (CPP paradigm) mNTS leptin reduced food-seeking but only under food-restricted conditions. Surprisingly, mNTS leptin signaling had no effect on learned appetitive behavior directed toward opiate reinforcement. These novel findings have implications, not only with respect to the leptinergic control of energy balance, but also regarding food intake control by mNTS neural processing more generally.

\section{MATERIALS AND METHODS}

\section{Subjects}

Adult male Sprague-Dawley rats (250-300 g upon arrival; Charles River Laboratories, Wilmington, MA), housed individually in hanging metal cages and maintained on a 12: 12-h light-dark cycle (lights off at 0900 hours for Exps 12; lights on at 0800 hours for Exps 3-4), had ad libitum access to rodent chow (5001 Rodent diet; Lab Diets, St Louis, MO) and water unless otherwise noted. All protocols and procedures conformed to the institutional standards of the University of Pennsylvania Animal Care and Use Committee and were approved by the committee.

\section{Surgery}

Rats were injected with ketamine $(90 \mathrm{mg} / \mathrm{kg}$; Bulter Animal Health Supply, Dublin, $\mathrm{OH})$, xylazine $(2.7 \mathrm{mg} / \mathrm{kg}$; Anased, Shenandoah, IA), and acepromazine $(0.64 \mathrm{mg} / \mathrm{kg} ;$ Bulter Animal Health Supply) anesthesia and analgesia $(2 \mathrm{mg} / \mathrm{kg}$ Metacam; Boehringer Ingelheim Vet-medica, St Joseph, $\mathrm{MO}$ ) for all surgeries. Bilateral guide cannulae (26-gauge; Plastics One, Roanoke, VA; $1.5 \mathrm{~mm}$ spacing between cannulae) were implanted with the tip stereotaxically positioned above the mNTS target injection site using one of two coordinate systems (coordinates varied by surgeon to maximize overall accuracy): (1) midline, $1.0 \mathrm{~mm}$ posterior to occipital crest, $6.7 \mathrm{~mm}$ ventral from skull surface, injection targeted $2.0 \mathrm{~mm}$ below end of guide cannula; or (2) midline, $2.0 \mathrm{~mm}$ anterior to occipital crest, $6.8 \mathrm{~mm}$ ventral from skull surface using a 15-degree angle (negative slope in anterior to posterior direction), injection targeted $1.5 \mathrm{~mm}$ below end of guide cannula. Procedures for unilateral mNTS injections (right side) were based on our previous work (eg, Hayes et al, 2009, 2011; Kanoski et al, 2012a, b; Spaeth et al, 2012) and involve $100 \mathrm{nl}$ infusion (delivery rate of $5 \mathrm{ml} / \mathrm{min}$ via Harvard Apparatus PHD 2000 infusion pump; 28 gauge indwelling injector cannulae) followed by a 30- to 40-s diffusion period where the injectors are left in place. Intended anatomic positions of mNTS injection sites were evaluated 1 week post-surgery by measurement of the sympathoadrenal-mediated glycemic response to an injection of 5-thio-D-glucose $(24 \mu \mathrm{g} / 100 \mathrm{nl}$ unilateral mNTS injection; Ritter et al, 1981). A postinjection elevation in baseline plasma glucose level of $100 \%$ or greater was required for subject inclusion (assessed via tail nick at baseline, $30 \mathrm{~min}$, and $60 \mathrm{~min}$ following 5TG infusion). Cannula placement was further confirmed 


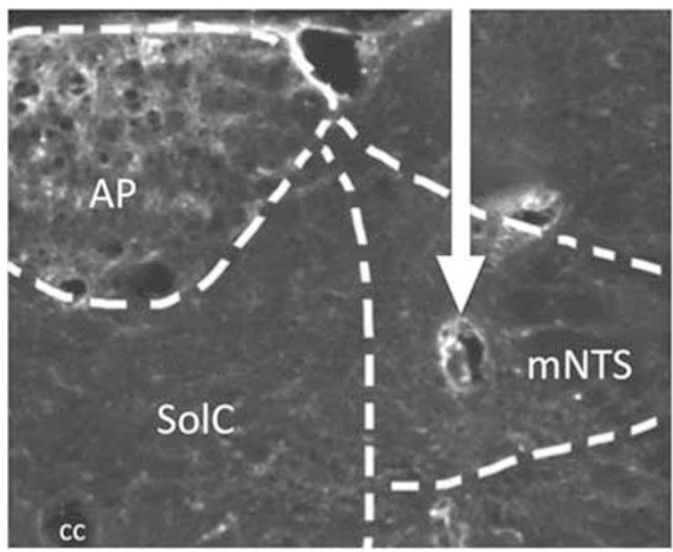

Figure I Representative mNTS injection site.

postmortem via bilateral histological location of $100 \mathrm{nl}$ volume injection of Chicago sky blue ink. A representative injection placement is depicted in the right mNTS in Figure 1 (Note: vehicle and leptin were administered unilaterally for all experiments). Animals without confirmation of functional and anatomical injection placement were removed from analyses. Overall, four animals were excluded from Exp. 2 analyses, four animals were excluded from Exp. 3 analyses, and five animals were excluded from Exp. 4 analyses.

\section{Procedures}

Experiment 1: free-feeding food intake assessment. Naive rats were unilaterally infused intra-mNTS unilaterally with $0.0 \mu \mathrm{g}$ (vehicle; sodium bicarbonate), $0.1,0.3$, or $0.6 \mu \mathrm{g}$ leptin (100 nl volume) immediately before dark onset using a within-subjects design. Treatments were separated by 2-3 days. The rats were individually housed in wire-bottom cages with an access hole to a cup containing powdered rodent chow (5001 Rodent diet; Lab Diets) that rested on an electronic scale. The food cup weight was monitored by computer software (LabView). This automated-feeding system tracks cumulative food intake analysis via custom software over each 24-h period without disturbing the natural-feeding cycle of the rats (ie, eliminates experimenter interruption).

Experiment \#2a: PR operant responding for sucrose in chronically food-restricted rats. Naive rats $(n=11)$ were given operant lever press training for sucrose reinforcement as previously described (Davis et al, 2011; Kanoski et al, 2013). Before training rats were given daily chow rations to gradually (over 5-7 days) reduce body weight to $85 \%$ of an ad libitum body weight established before training. Daily rations were given throughout training and testing to maintain this $85 \%$ body weight criterion. Training was carried out over 6 days with a 1-h session each day in conditioning boxes (Med Associates; MedPC IV Software, St Albans, VT). During the first 2 days, a fixed ratio autoshaping procedure was used where each lever press earned a 45-mg sucrose pellet (Bio-Serv, Frenchtown, NJ) (FR1); a free sucrose pellet was also dispensed for every $600 \mathrm{~s}$ that elapsed without operant-based reinforcement. The animals then received 2 days of an FR1 schedule with no autoshaping component followed by 2 days of FR3 training. For all procedures, the right lever was the 'active' lever; a left 'inactive' lever served as a control (dummy lever) for non-conditioned elevations in responding.

The rats were given two tests (within-subjects design, 2 rest days intervened between tests) using a PR reinforcement schedule. The unilateral mNTS injections (100 nl vehicle or $0.3 \mu \mathrm{g}$ leptin; order counterbalanced) were given $3 \mathrm{~h}$ before each test session. The 3-h timing (used for Exps 2-4) was chosen based on results of Exp. 1 showing this dose to be the lowest effective dose for significant food intake suppression $3 \mathrm{~h}$ after injections. After injections, the animals were returned to their home cages for the 3-h time period between injections and $\mathrm{PR}$ testing. The response requirement of the $\mathrm{PR}$ schedule increased progressively as previously described (Davis et al, 2011; Kanoski et al, 2013), using the following formula: $F(i)=5 e^{\wedge} 0.2 i-5$, where $F(i)$ is the number of lever presses required for next pellet at $\mathrm{i}=$ pellet number. The breakpoint for each animal was defined as the final completed lever press requirement that preceded a 20 -min period without earning a reinforcer.

Experiment \#2b: PR operant responding for sucrose in ad libitum-fed rats. Exp. 2b was conducted in a separate group of rats $(n=11)$. Procedures were identical to Exp. 2a, except that the rats were not food restricted (ad libitum access to chow in home cage) throughout the entire experiment, except during the 3 -h period between mNTS injections and PR testing when food was removed from the home cage.

Experiment \#3a: CPP for food reward in chronically foodrestricted rats. Before and throughout training and testing, naive rats $(n=13)$ were given the $85 \%$ body weight food restriction regime described in Exp. 2a. The procedures for appetitive CPP were as we have previously described (Kanoski et al, 2011). All CPP training and testing procedures were conducted in a dimly lit room. The procedures were conducted in two identical conjoined plexiglass CPP compartments $(74 \mathrm{~cm}$ long, $57.4 \mathrm{~cm}$ wide, and $24.7 \mathrm{~cm}$ wall height) with a removable divider wall in the center. The two sides (henceforth referred to as contexts or locations) of the CPP chamber were made distinguishable by varying wall color (white vs black plexiglass), floor texture (plexiglass vs steel grid), and orientation of stripes (adhesive tape applied vertically $v s$ horizontally) on the walls. Rats were first allowed to freely explore the CPP chamber during one 10-min habituation session in which the divider wall was removed and the time spent in each of the two contexts was recorded by an experimenter. For each rat, the context that was least preferred during this habituation session was assigned as the food-paired context for subsequent training, whereas the more preferred side was never paired with food. CPP training consisted of six, 20-min sessions (one session per day): three sessions where the rat was isolated in the food-paired context and three sessions isolated in the nonfood-paired context. During food-paired training sessions, a total of $5 \mathrm{~g}$ of a high-fat/ high-sucrose diet (Research Diets; D12492) was divided into five aliquots that were scattered throughout the context, whereas no food was given during nonfood-paired sessions. 
All rats consumed the entire $5 \mathrm{~g}$ during each food-paired session. The training order was randomized and pairmatched across groups. The first three and last three sessions occurred on continuous days; sessions 1-3 and 4-6 were separated by two intervening days.

Testing occurred 2 days after the sixth training session using a between-subjects design. Rats were assigned to groups matched for baseline context preference $(n=7$ for leptin, $n=6$ for vehicle). For each rat, a unilateral mNTS injection of either vehicle or leptin $(0.3 \mu \mathrm{g})$ was given $3 \mathrm{~h}$ before a 15-min video-recorded test session in which the center divider was removed and no food was given. The rats were returned to the home cage for the $3 \mathrm{~h}$ between injections and CPP testing. The time spent in each context during the test was later calculated from analysis of the video recording made by an experimenter blind to the group assignments and context food assignments. The dependent variable used for analysis was the percentage shift in preference score for the food-associated context from the baseline session compared with the test session.

Experiment \#3b: CPP for food reward in ad libitum-fed rats. Our pilot work (data not shown) showed that using the same training regime as Exp. 3a resulted in unreliable establishment of CPP in non-restricted (ad libitum fed) rats using the same training regime as Exp. 4. Thus, the number of training sessions was increased from 6 ( 3 per context) to 16 (8 per context) to ensure baseline shift in CPP in vehicletreated ad libitum-fed rats. All other procedures were identical to Exp. 4 except that the rats were not food restricted at any point throughout the experiment (except for during the 3-h period between mNTS injections and CPP testing). Four training sessions were given each week with no more than two intervening days between sessions.

Experiment \#4a: CPP for morphine reward in chronically food-restricted rats. Naive rats $(n=18)$ were maintained on $85 \%$ food restriction regime as described for Exps 2a and $3 a$. The procedures for morphine CPP were modified from Harris et al (2007) and used the came CPP apparatuses as Exp. 3. A 15-min habituation session was first conducted as described for Exp. 3, and the morphine-associated side was assigned for each rat based on their least preferred location during the habituation session. Three days later, training began and continued across 3 consecutive training days. For each training day, rats were given IP injections of either saline (vehicle) or morphine $(10 \mathrm{mg} / \mathrm{kg})$ immediately before being confined to the appropriately assigned compartment for $30 \mathrm{~min}$. Morphine and saline sessions alternated between the morning and afternoon with a 4 -h interval between sessions. Rats given morphine before the morning training session were given saline before being placed in the opposite chamber in the afternoon, and on subsequent days received saline in the morning and morphine in the afternoon (ABA order). The number of rats in which the morning training session on the first training day involved morning saline $v s$ morphine injections was counterbalanced across the two drug groups. Two days after the last training session, the rats received either intra-mNTS unilateral vehicle or leptin $(0.3 \mu \mathrm{g})$ injections $3 \mathrm{~h}$ before a 15 -min

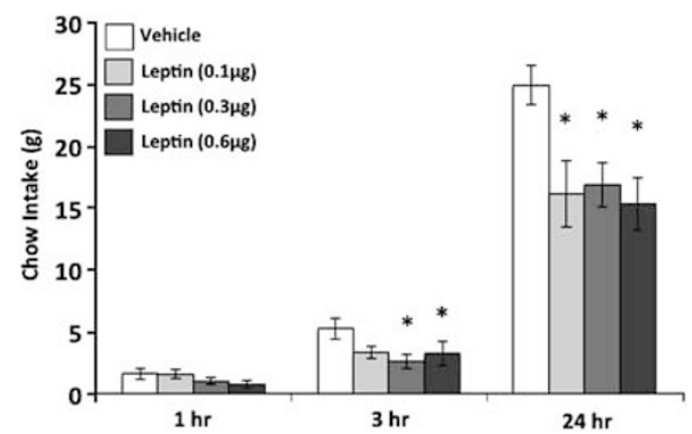

Figure 2 Cumulative home cage food intake in non-restricted rats following mNTS leptin administration (means $\pm \mathrm{SEM}$; *indicates $P$-value $<0.0 \mathrm{I}$ ).

CPP test in which no prior IP morphine or saline injections were given and the divider wall was removed. Drug groups ( $n=8$ for vehicle, $n=10$ for leptin) were assigned according to baseline preference and body weight. The amount of time spent in each of the two separate contexts during testing was calculated via analysis of video recording by an experimenter blind to the treatment groups and the assigned drug locations.

Experiment \#4b: CPP for morphine reward in ad libitumfed rats. A separate group of naive rats $(n=18)$ were separated into vehicle or morphine (IP; $10 \mathrm{mg} / \mathrm{kg} /$ day) groups ( $n=9$ per group) and underwent the same procedures as Exp. 4a except they had ad libitum access to rat chow (except for the 3 -h period between mNTS leptin injection and CPP testing).

\section{Data and Statistical Analyses}

Data from Exp. 1 were analyzed via ANOVA using drug treatment as a repeated-measure variable. When significant overall main effects of drug were found, individual drug dose treatments were compared with vehicle treatment using Tukey's post hoc comparisons. Repeated-measures ANOVA was also used to compare drug conditions in the PR experiments (Exps 2a and 2b), whereas one-way ANOVA was used for the between-subjects CPP experiments (3a, 3b, 4a, and 4b). Alpha level for significance was set at 0.05 for all described experiments.

\section{RESULTS}

Exp. 1: mNTS Leptin Administration Reduces Chow Intake in Free-Feeding Rats

The two higher doses of leptin $(0.3$ and $0.6 \mu \mathrm{g})$ suppressed cumulative chow intake relative to vehicle at $3 \mathrm{~h}$ after drug administration, whereas all three doses suppressed intake at $24 \mathrm{~h}$ (Figure 2). The main effect of leptin was significant at $3 \mathrm{~h} \quad(\mathrm{~F}(3,24)=5.08, \quad P<0.01)$ and $24 \mathrm{~h} \quad(\mathrm{~F}(3,24)=5.01$, $P<0.01)$. Although the main effect of leptin was also significant at $1 \mathrm{~h}(\mathrm{~F}(3,24)=3.27, P<0.05)$, no individual leptin treatment significantly differed from vehicle in the post hoc Tukey's comparisons. 

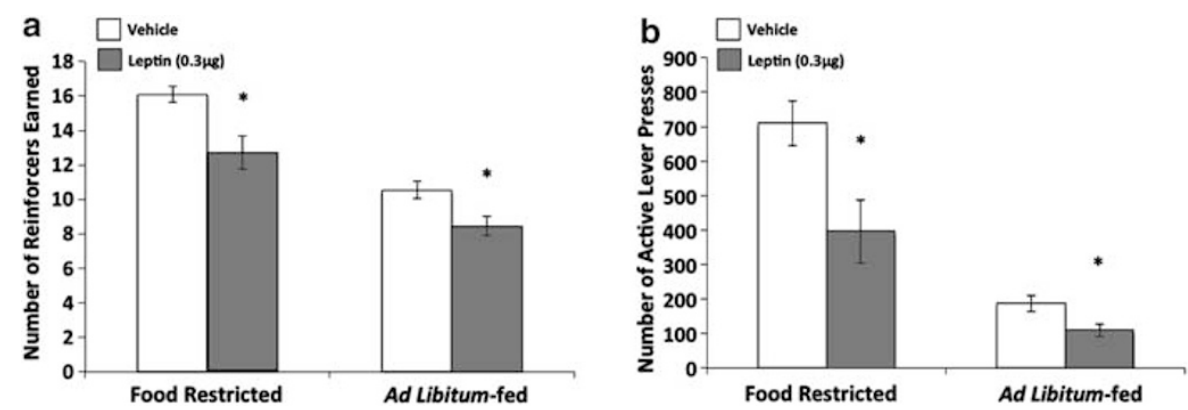

Figure 3 Operant lever pressing for sucrose in a progressive ratio reinforcement test for both food-restricted and non-restricted rats, shown as: (a) the number of reinforcers ( $45 \mathrm{mg}$ sucrose pellets) earned before reaching breakpoint criterion, and (b) the number of active lever presses performed before reaching breakpoint criterion (means $\pm \mathrm{SEM}$; *indicates $P$-value $<0.0 \mathrm{I}$ ).

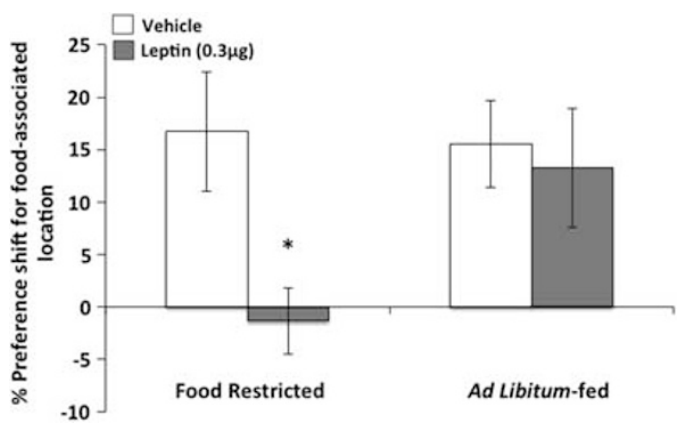

Figure 4 Conditioned place preference expression for an environment previously associated with consuming palatable food (high fat and high sucrose) for both food-restricted and non-restricted rats (means \pm SEM; *indicates $P$-value $<0.0 \mathrm{I}$ ).

Exps 2a and 2b: mNTS Leptin Administration Reduces Operant Responding for Sucrose in Both Chronically Food-Restricted and Free-Feeding Rats

Leptin $(0.3 \mu \mathrm{g})$ delivered to the mNTS $3 \mathrm{~h}$ before testing significantly suppressed the breakpoint for lever press responding for sucrose under a PR schedule for both foodrestricted (Exp. 2a) and non-restricted, ad libitum-fed rats (Exp. 2b). This conclusion is supported by significant drug effects for the number of reinforcers earned before breakpoint (Figure 3a), $(\mathrm{F}(1,10)=10.50, P<0.01$ for Exp. 2a; $\mathrm{F}(1,10)=11.21, P<0.01$ for Exp. $2 \mathrm{~b}$ ), the breakpoint (data not shown), $(\mathrm{F}(1,10)=10.97, \quad P<0.01$ for Exp. 2a; $\mathrm{F}(1,10)=9.65, P<0.05$ for Exp. 2b), and the number of lever presses on the active lever before breakpoint (Figure 3b), $\quad(\mathrm{F}(1,10)=9.33, \quad P<0.05$ for Exp. 2a; $\mathrm{F}(1,10)=7.68, P<0.05$ for Exp. 2b). For both Exps 2a and $2 \mathrm{~b}$, the number of lever presses on the inactive control lever was not influenced by mNTS leptin delivery (data not shown), $(\mathrm{F}(1,10)<3.17, P>0.1)$.

\section{Exps 3a and 3b: CPP for Food Reward is Reduced by} mNTS Leptin Delivery in Food-Restricted, but Not $A d$ Libitum-Fed Rats

Unlike vehicle-treated rats, food-restricted rats infused intra-mNTS $\mathrm{w} / 0.3 \mu \mathrm{g}$ leptin before CPP testing did not display a shift in preference from baseline (before training) for the food-associated environment (Exp. 3a; Figure 4).

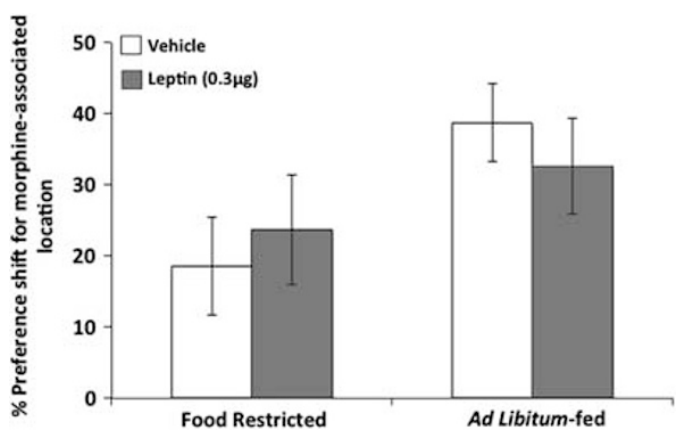

Figure 5 Conditioned place preference expression for a location previously associated with morphine reinforcement for both foodrestricted and non-restricted rats (means \pm SEM).

However, mNTS leptin delivery did not impact the expression of the food reward CPP relative to vehicle treatment for non-restricted rats (Exp. 3b; Figure 4). The main effect of drug was significant for CPP testing in Exp. 3a $(\mathrm{F}(1,11)=8.36, P<0.05)$, but not for Exp. 3b $(\mathrm{F}(1,23)=0.10)$.

\section{Exps 4a and 4b: CPP for Morphine-Associated} Environment is Not Affected by mNTS LepRb Activation

Relative to vehicle treatment, $0.3 \mu \mathrm{g}$ leptin delivery to the mNTS did not impact the expression of CPP for an environment associated with morphine reinforcement (Figure 5). This was the case for both food restricted (Exp. 4a), $(\mathrm{F}(1,16)=0.23)$, and non-restricted rats (Exp. $4 \mathrm{~b}),(\mathrm{F}(1,16)=0.50)$.

\section{DISCUSSION}

Leptin's anorectic effects are mediated, in part, by amplifying the food intake-suppressive effects of GI satiation signals via LepRb signaling in mNTS neurons (Hayes et al, 2010; Huo et al, 2007; Kanoski et al, 2012b). Here we highlight a novel role for mNTS LepRb signaling in the suppression of appetitive responding for palatable food. Results revealed reduced food-motivated responding following mNTS LepRb activation in two appetitive behavioral tests: (1) operant lever pressing for sucrose under a PR 
reinforcement schedule, and (2) the expression of a CPP for palatable (high fat and high sucrose) food. The pattern of these effects were dependent on: (1) the presence $v s$ absence of food consumption during testing, (2) the long-term energy status of the animals (chronic food restriction $v s$ ad libitum-fed), and (3) nature of the positive reinforcement (palatable food $v s$ morphine). Each of these features of our findings is discussed below.

Effort-based responding for sucrose in the PR leverpressing test was reduced by mNTS LepRb activation regardless of whether the rats were chronically food restricted (Exp. 2a) or ad libitum fed (Exp. 2b). The PR breakpoint test conditions allowed for periodic consumption of the reinforcement after the exponentially increasing operant lever press demands were met (totaling approximately $0.33-0.75 \mathrm{~g}$ consumed per rat). Thus, effects on motivation to obtain the reward cannot be separated in terms of appetitive/incentive $v s$ consummatory aspects of the reinforcement. In this sense, the reduced lever press responding for sucrose observed following mNTS LepRb activation can be interpreted as being attributable to effects on orosensory or postingestive processing (eg, amplifying satiation signals or altering taste) as opposed to effects on appetitive, food-seeking behavior per se. However, the case for suppression of appetitive behavior independent of orosensory or postingestive processing is supported by Exp. 3a results (CPP in food-restricted rats). In this case, CPP expression for food reward was suppressed by mNTS leptin despite the fact that the animals received no food during the 24-h period preceding CPP testing, nor did they receive food during the $\mathrm{CPP}$ test. In addition, these findings highlight a potential CNS site of action contributing to the inhibition of food-seeking/hoarding previously observed in food-restricted hamsters treated with peripheral or ICV leptin (Buckley and Schneider, 2003; Keen-Rhinehart and Bartness, 2008).

Unlike results from PR lever-pressing tests, CPP for food reward was not influenced by mNTS LepRb activation in animals that were maintained on an ad libitum feeding schedule and were only food restricted for $3 \mathrm{~h}$ before and during CPP testing (Exp. 3b). The differential effect of energy status on mNTS leptin-induced inhibition of lever pressing vs CPP may be based, in part, on the different underlying neural substrates between these two behavioral tests. Unlike the PR test, CPP expression requires hippocampal-dependent memory retrieval of external contextual cues. In addition to processing external contextual cues, neural processing in the hippocampus is also required for encoding the relationships between internal states (eg, different levels of food deprivation), the external environment, and goal-directed behavior (Kennedy and Shapiro, 2004). It may be that mNTS LepRb activation inhibits hippocampal-dependent contextual memory retrieval via an ascending polysynaptic pathway (eg, Castle et al, 2005), and that activation of this pathway is dependent on internal context (upregulated during energy restriction).

Alternatively, the different pattern of PR $v s \mathrm{CPP}$ results may be based on the fact that these two tests differ with regards to the opportunity for the animals to consume the food. For example, mNTS LepRb signaling reduces motivated responding for food reward independent of energy status when orosensory and postingestive contact with food occurs (PR lever-pressing schedule; Exps 2a and 2b), suggesting that leptin signaling in this brain region may augment postingestive satiation signaling in both an energydeplete and -replete state. However, in the absence of food intake (CPP for food; Exps 3a and $3 b$ ), LepRb activation in the mNTS only reduces motivated responding for food in an energy-deplete state (chronic food restriction). According to Rosenbaum and Leibel (2011), when energy stores drop below a neurally encoded threshold for minimum body fat, a compensatory physiological drive is invoked to help restore adipose stores. Within this framework, low endogenous LepRb activation on mNTS neurons in states of chronic food restriction may serve as a neural signal to engage in food-seeking behavior, and this behavior is suppressed when leptin levels are normalized endogenously (weight or adipose gain) or pharmacologically via mNTS exogenous delivery (Exp. 3a), whereas food seeking that occurs in the absence of metabolic need (Exp. 3b) may be less influenced by mNTS leptin signaling.

Unlike CPP for food reinforcement, CPP for morphine reinforcement was not affected by mNTS LepRb activation regardless of whether the animals were food restricted or maintained on ad libitum feeding. These results were unexpected for several reasons. First, CNS-delivered leptin (forebrain ventricle) reduces self-administration of rewarding electrical stimulation of the lateral hypothalamic area (LHA) in rats (Fulton et al, 2000, 2004), suggesting that CNS leptin may reduce reward value more generally. Consistent with these findings, leptin acts on receptors in the VTA to modulate meso-accumbal dopamine transmission and reduce feeding (Baldo and Kelley, 2007; Fulton et al, 2006; Hommel et al, 2006). One interpretation is that leptin action on receptors in certain forebrain and midbrain nuclei, such as the LHA and VTA, suppresses motivated responding for a range of reinforcers, whereas mNTS LepRb signaling is more specific to food reinforcement. However, there is evidence that LepRb and opioid receptor signaling interact in mNTS neurons based on findings that these systems have opposing effects on vagally mediated excitatory afferent drive. Neurophysiological data from Appleyard, Peters, and others show that while opioid agonists reduce the excitatory vagal afferent drive on mNTS neurons (Appleyard et al, 2005; Cui et al, 2012), leptin activates cultured nodose ganglia neurons in a cooperative manner with CCK (Peters et al, 2004, 2005, 2006). Further, peripheral administration of naloxone, an opioid receptor antagonist, decreases meal size in food-restricted sucrose-fed rats (Glass et al, 2001), an effect also observed following CNS or peripheral LepRb agonist delivery (Eckel et al, 1998; Kahler et al, 1998; Zorrilla et al, 2005). Thus, although leptin and opioid receptor activation appear to interact by differentially modulating GI-derived satiation signaling, our CPP results suggest that with regards to appetitive responding, mNTS LepRb signaling may have selective effects on reducing motivation to obtain food reinforcement. However, we note that this hypothesis requires testing across a range of other appetitive reinforcements. An alternative interpretation is that the differential effects of mNTS LepRb activation on appetitive responding for food $v s$ opioid reinforcement is based on differences in reward magnitude. In other words, the putative higher reward magnitude of morphine 
compared with food reinforcement may account for the differential effects of mNTS leptin on CPP responding. This interpretation is unlikely, however, given that mNTS leptin reduced CPP for food but not morphine in food-restricted rats despite the fact that the parameters used for CPP training and testing in these two experiments (Exps $3 \mathrm{a}$ and 4a) yielded comparable preference shifts following vehicle administration.

Overall results revealed that mNTS LepRb activation reduced food-seeking behavior and effort-based responding for palatable food. These findings alter the conventional wisdom that learned incentive and motivational aspects of feeding are uniquely controlled by neural processing in mesencephalon, diencephalon, and telencephalon regions. Mesolimbic dopaminergic signaling from dopamine-producing VTA neurons to ventral striatal nuclei is generally considered to be a central neural system in controlling incentive and motivational components of ingestion (Baldo and Kelley, 2007; Berridge, 2007; Kelley, 2004; Kenny, 2011; Volkow et al, 2011). Indeed, both CPP and operant lever pressing for food reward are modulated by manipulations in striatal DA receptor activity (for review, see Baldo et al, 2013). Our results suggest that foodseeking behavior also involves neuroendocrine signaling in the hindbrain, which likely engages ascending neural communication to mesolimbic nuclei that are traditionally linked with appetitive control. Consistent with this notion, ascending neural pathways have been characterized from the mNTS to the VTA (Rinaman, 2010), as well as from the mNTS to the nucleus accumbens (Alhadeff et al, 2012; Shekhtman et al, 2007). The role of these ascending pathways in the suppression of appetitive responding by mNTS LepRb signaling remains to be determined. A related area for future mechanistic follow-up work is to delineate the intracellular and downstream neurochemical mediators of mNTS LepRb-mediated inhibition of foodmotivated behavior. Recent data reveal that GLP-1-producing (preproglucagon (PPG)-expressing) neurons in the mNTS project to the VTA and nucleus accumbens where GLP-1 receptor (GLP1-R) activity modulates feeding and food-motivated responding (Alhadeff et al, 2012; Dickson et al, 2012; Dossat et al, 2011). It may be that LepRb activation on mNTS neurons augments PPG neural transmission to mesolimbic GLP-1R-expressing nuclei via either direct (PPG and LepRb are co-expressed in mNTS neurons for the mouse (Garfield et al, 2012; Goldstone et al, 1997; Huo et al, 2008)) or indirect pathways (PPG and LepRb are not co-expressed in mNTS neurons for the rat; Huo et al, 2008).

\section{FUNDING AND DISCLOSURE}

The authors declare no conflict of interest.

\section{ACKNOWLEDGEMENTS}

We thank Rick Ritacco, Kalina Eneva, Matthew Hayes, and Katie Ricks for substantial contributions. This work was funded by NIH-DK21397 (HJG) and NIHDK089752 (SEK).

\section{REFERENCES}

Ahima RS, Saper CB, Flier JS, Elmquist JK (2000). Leptin regulation of neuroendocrine systems. Front Neuroendocrinol 21: 263-307.

Alhadeff AL, Rupprecht LE, Hayes MR (2012). GLP-1 Neurons in the nucleus of the solitary tract project directly to the ventral tegmental area and nucleus accumbens to control for food intake. Endocrinology 153: 647-658.

Appleyard SM, Bailey TW, Doyle MW, Jin YH, Smart JL, Low MJ et al (2005). Proopiomelanocortin neurons in nucleus tractus solitarius are activated by visceral afferents: regulation by cholecystokinin and opioids. J Neurosci 25: 3578-3585.

Baldo BA, Kelley AE (2007). Discrete neurochemical coding of distinguishable motivational processes: insights from nucleus accumbens control of feeding. Psychopharmacology (Berl) 191: 439-459.

Baldo BA, Pratt WE, Will MJ, Hanlon EC, Bakshi VP, Cador M (2013). Principles of motivation revealed by the diverse functions of neuropharmacological and neuroanatomical substrates underlying feeding behavior. Neurosci Biobehav Rev (e-pub ahead of print).

Berridge KC (2007). The debate over dopamine's role in reward: the case for incentive salience. Psychopharmacology (Berl) 191: 391-431.

Buckley CA, Schneider JE (2003). Food hoarding is increased by food deprivation and decreased by leptin treatment in Syrian hamsters. Am J Physiol Regul Integr Comp Physiol 285: R1021-R1029.

Caron E, Sachot C, Prevot V, Bouret SG (2010). Distribution of leptin-sensitive cells in the postnatal and adult mouse brain. J Comp Neurol 518: 459-476.

Castle M, Comoli E, Loewy AD (2005). Autonomic brainstem nuclei are linked to the hippocampus. Neuroscience 134: 657-669.

Cui RJ, Roberts BL, Zhao H, Andresen MC, Appleyard SM (2012). Opioids inhibit visceral afferent activation of catecholamine neurons in the solitary tract nucleus. Neuroscience 222: 181-190.

Davis JF, Choi DL, Schurdak JD, Fitzgerald MF, Clegg DJ, Lipton JW et al (2011). Leptin regulates energy balance and motivation through action at distinct neural circuits. Biol Psychiatry 69: 668-674.

De Luca MA, Bimpisidis Z, Bassareo V, Di Chiara G (2011). Influence of morphine sensitization on the responsiveness of mesolimbic and mesocortical dopamine transmission to appetitive and aversive gustatory stimuli. Psychopharmacology (Berl) 216: 345-353.

Dickson SL, Shirazi RH, Hansson C, Bergquist F, Nissbrandt H, Skibicka KP (2012). The glucagon-like peptide 1 (GLP-1) analogue, exendin-4, decreases the rewarding value of food: a new role for mesolimbic GLP-1 receptors. J Neurosci 32: 4812-4820.

Dossat AM, Lilly N, Kay K, Williams DL (2011). Glucagon-like peptide 1 receptors in nucleus accumbens affect food intake. J Neurosci 31: 14453-14457.

Eckel LA, Langhans W, Kahler A, Campfield LA, Smith FJ, Geary N (1998). Chronic administration of OB protein decreases food intake by selectively reducing meal size in female rats. Am J Physiol 275(1 Pt 2): R186-R193.

Elias CF, Kelly JF, Lee CE, Ahima RS, Drucker DJ, Saper CB et al (2000). Chemical characterization of leptin-activated neurons in the rat brain. J Comp Neurol 423: 261-281.

Fulton S, Pissios P, Manchon RP, Stiles L, Frank L, Pothos EN et al (2006). Leptin regulation of the mesoaccumbens dopamine pathway. Neuron 51: 811-822.

Fulton S, Richard D, Woodside B, Shizgal P (2004). Food restriction and leptin impact brain reward circuitry in lean and obese Zucker rats. Behav Brain Res 155: 319-329.

Fulton S, Woodside B, Shizgal P (2000). Modulation of brain reward circuitry by leptin. Science 287: 125-128. 
Garfield AS, Patterson C, Skora S, Gribble FM, Reimann F, Evans ML et al (2012). Neurochemical characterization of body weightregulating leptin receptor neurons in the nucleus of the solitary tract. Endocrinology 153: 4600-4607.

Glass MJ, Grace MK, Cleary JP, Billington CJ, Levine AS (2001). Naloxone's effect on meal microstructure of sucrose and cornstarch diets. Am J Physiol Regul Integr Comp Physiol 281: R1605-R1612.

Goldstone AP, Mercer JG, Gunn I, Moar KM, Edwards CM, Rossi M et al (1997). Leptin interacts with glucagon-like peptide-1 neurons to reduce food intake and body weight in rodents. FEBS Lett 415: 134-138.

Grill HJ (2010). Leptin and the systems neuroscience of meal size control. Front Neuroendocrinol 31: 61-78.

Grill HJ, Hayes MR (2012). Hindbrain neurons as an essential hub in the neuroanatomically distributed control of energy balance. Cell Metab 16: 296-309.

Grill HJ, Hayes MR (2009). The nucleus tractus solitarius: a portal for visceral afferent signal processing, energy status assessment and integration of their combined effects on food intake. Int $J$ Obes (Lond) 33(Suppl 1): S11-S15.

Grill HJ, Kaplan JM (1990). In: Stricker EM, Edward M (eds). Caudal Brainstem Participates in the Distributed Neural Control of Feeding. Plenum Press: NY, USA.

Grill HJ, Kaplan JM (2002a). The neuroanatomical axis for control of energy balance. Front Neuroendocrinol 23: 2-40.

Grill HJ, Schwartz MW, Kaplan JM, Foxhall JS, Breininger J, Baskin DG (2002b). Evidence that the caudal brainstem is a target for the inhibitory effect of leptin on food intake. Endocrinology 143: 239-246.

Harris GC, Wimmer M, Randall-Thompson JF, Aston-Jones G (2007). Lateral hypothalamic orexin neurons are critically involved in learning to associate an environment with morphine reward. Behav Brain Res 183: 43-51.

Hayes MR, Bradley L, Grill HJ (2009). Endogenous hindbrain glucagon-like peptide-1 receptor activation contributes to the control of food intake by mediating gastric satiation signaling. Endocrinology 150: 2654-2659.

Hayes MR, Leichner TM, Zhao S, Lee GS, Chowansky A, Zimmer D et al (2011). Intracellular signals mediating the food intakesuppressive effects of hindbrain glucagon-like peptide-1 receptor activation. Cell Metab 13: 320-330.

Hayes MR, Skibicka KP, Leichner TM, Guarnieri DJ, DiLeone RJ, Bence KK et al (2010). Endogenous leptin signaling in the caudal nucleus tractus solitarius and area postrema is required for energy balance regulation. Cell Metab 11: 77-83.

Hommel JD, Trinko R, Sears RM, Georgescu D, Liu ZW, Gao XB et al (2006). Leptin receptor signaling in midbrain dopamine neurons regulates feeding. Neuron 51: 801-810.

Huo L, Gamber KM, Grill HJ, Bjorbaek C (2008). Divergent leptin signaling in proglucagon neurons of the nucleus of the solitary tract in mice and rats. Endocrinology 149: 492-497.

Huo L, Grill HJ, Bjorbaek C (2006). Divergent regulation of proopiomelanocortin neurons by leptin in the nucleus of the solitary tract and in the arcuate hypothalamic nucleus. Diabetes 55: 567-573.

Huo L, Maeng L, Bjorbaek C, Grill HJ (2007). Leptin and the control of food intake: neurons in the nucleus of the solitary tract are activated by both gastric distension and leptin. Endocrinology 148: 2189-2197.

Kahler A, Geary N, Eckel LA, Campfield LA, Smith FJ, Langhans W (1998). Chronic administration of OB protein decreases food intake by selectively reducing meal size in male rats. $A m J$ Physiol 275(1 Pt 2): R180-R185.

Kanoski SE, Fortin SM, Ricks KM, Grill HJ (2013). Ghrelin signaling in the ventral hippocampus stimulates learned and motivational aspects of feeding via PI3K-Akt signaling. Biol Psychiatry 73: 915-923.
Kanoski SE, Hayes MR, Greenwald HS, Fortin SM, Gianessi CA, Gilbert JR et al (2011). Hippocampal leptin signaling reduces food intake and modulates food-related memory processing. Neuropsychopharmacology 36: 1859-1870.

Kanoski SE, Rupprecht LE, Fortin SM, De Jonghe BC, Hayes MR (2012a). The role of nausea in food intake and body weight suppression by peripheral GLP-1 receptor agonists, exendin-4 and liraglutide. Neuropharmacology 62: 1916-1927.

Kanoski SE, Zhao S, Guarnieri DJ, Dileone RJ, Yan J, De Jonghe BC et al (2012b). Endogenous leptin receptor signaling in the medial nucleus tractus solitarius affects meal size and potentiates intestinal satiation signals. Am J Physiol Endocrinol Metab 303: E496-E503.

Keen-Rhinehart E, Bartness TJ (2008). Leptin inhibits fooddeprivation-induced increases in food intake and food hoarding. Am J Physiol Regul Integr Comp Physiol 295: R1737-R1746.

Kelley AE (2004). Ventral striatal control of appetitive motivation: role in ingestive behavior and reward-related learning. Neurosci Biobehav Rev 27: 765-776.

Kennedy PJ, Shapiro ML (2004). Retrieving memories via internal context requires the hippocampus. J Neurosci 24: 6979-6985.

Kenny PJ (2011). Reward mechanisms in obesity: new insights and future directions. Neuron 69: 664-679.

Leinninger GM, Jo YH, Leshan RL, Louis GW, Yang H, Barrera JG et al (2009). Leptin acts via leptin receptor-expressing lateral hypothalamic neurons to modulate the mesolimbic dopamine system and suppress feeding. Cell Metab 10: 89-98.

Li HY, Wang LL, Yeh RS (1999). Leptin immunoreactivity in the central nervous system in normal and diabetic rats. Neuroreport 10: 437-442.

Munzberg H, Bjornholm M, Bates SH, Myers MG Jr. (2005). Leptin receptor action and mechanisms of leptin resistance. Cell Mol Life Sci 62: 642-652.

Patterson CM, Leshan RL, Jones JC, Myers MG Jr. (2011). Molecular mapping of mouse brain regions innervated by leptin receptor-expressing cells. Brain Res 1378: 18-28.

Peters JH, Karpiel AB, Ritter RC, Simasko SM (2004). Cooperative activation of cultured vagal afferent neurons by leptin and cholecystokinin. Endocrinology 145: 3652-3657.

Peters JH, McKay BM, Simasko SM, Ritter RC (2005). Leptininduced satiation mediated by abdominal vagal afferents. Am J Physiol Regul Integr Comp Physiol 288: R879-R884.

Peters JH, Ritter RC, Simasko SM (2006). Leptin and CCK modulate complementary background conductances to depolarize cultured nodose neurons.. Am J Physiol Cell Physiol 290: C427-C432.

Rinaman L (2010). Ascending projections from the caudal visceral nucleus of the solitary tract to brain regions involved in food intake and energy expenditure. Brain Res 1350: 18-34.

Ritter RC, Slusser PG, Stone S (1981). Glucoreceptors controlling feeding and blood glucose: Location in the hindbrain. Science 213: 451-453.

Rosenbaum ML, Leibel R (2011). Brain Reorganization following Weight Loss. In: Drewnowski AR, BJ (ed) Obesity Treatment and Prevention: New Directions. Vol. 73 (Karger: Seattle, WA, USA), pp 1-20.

Schwartz GJ (2010). Brainstem integrative function in the central nervous system control of food intake. Forum Nutr 63: 141-151.

Schwartz GJ, Moran TH (2002). Leptin and neuropeptide y have opposing modulatory effects on nucleus of the solitary tract neurophysiological responses to gastric loads: implications for the control of food intake. Endocrinology 143: 3779-3784.

Shalev U, Yap J, Shaham Y (2001). Leptin attenuates acute food deprivation-induced relapse to heroin seeking. J Neurosci 21: RC129.

Shekhtman E, Geerling JC, Loewy AD (2007). Aldosteronesensitive neurons of the nucleus of the solitary tract: multisynaptic pathway to the nucleus accumbens. J Comp Neurol 501: 274-289. 
Skibicka KP, Grill HJ (2009). Hindbrain leptin stimulation induces anorexia and hyperthermia mediated by hindbrain melanocortin receptors. Endocrinology 150: 1705-1711.

Spaeth AM, Kanoski SE, Hayes MR, Grill HJ (2012). TrkB receptor signaling in the nucleus tractus solitarius mediates the food intake-suppressive effects of hindbrain BDNF and leptin. Am J Physiol Endocrinol Metab 302: E1252-E1260.

Tomasi D, Volkow ND (2013). Striatocortical pathway dysfunction in addiction and obesity: differences and similarities. Crit Rev Biochem Mol Biol 48: 1-19.

Volkow ND, Wang GJ, Baler RD (2011). Reward, dopamine and the control of food intake: implications for obesity. Trends Cognitive Sci 15: 37-46.
Volkow ND, Wang GJ, Tomasi D, Baler RD (2013a). The addictive dimensionality of obesity. Biol Psychiatry 73: 811-818.

Volkow ND, Wang GJ, Tomasi D, Baler RD (2013b). Obesity and addiction: neurobiological overlaps. Obes Rev 14: 2-18.

Zhang Y, Kerman IA, Laque A, Nguyen P, Faouzi M, Louis GW et al (2011). Leptin-receptor-expressing neurons in the dorsomedial hypothalamus and median preoptic area regulate sympathetic brown adipose tissue circuits. $J$ Neurosci 31: 1873-1884.

Zorrilla EP, Inoue K, Valdez GR, Tabarin A, Koob GF (2005). Leptin and post-prandial satiety: acute central leptin more potently reduces meal frequency than meal size in the rat. Psychopharmacology (Berl) 177: 324-335. 